\title{
Symmetry Analysis for a Fourth-order Noise-reduction Partial Differential Equation
}

\author{
Andronikos Paliathanasis* \\ Institute of Systems Science, Durban University of Technology \\ PO Box 1334, Durban 4000, Republic of South Africa \\ Instituto de Ciencias Físicas y Matemáticas, \\ Universidad Austral de Chile, Valdivia, Chile \\ P.G.L. Leach \\ Institute of Systems Science, Durban University of Technology \\ PO Box 1334, Durban 4000, Republic of South Africa \\ School of Mathematical Sciences, University of KwaZulu-Natal \\ Durban, Republic of South Africa
}

August 17, 2020

\begin{abstract}
We apply the theory of Lie symmetries in order to study a fourth-order $1+2$ evolutionary partial differential equation which has been proposed for the image processing noise reduction. In particular we determine the Lie point symmetries for the specific $1+2$ partial differential equations and we apply the invariant functions to determine similarity solutions. For the static solutions we observe that the reduced fourth-order ordinary differential equations are reduced to second-order ordinary differential equations which are maximally symmetric. Finally, nonstatic closed-form solutions are also determined.
\end{abstract}

Keywords: Lie symmetries, Noise reduction, Image processing

\section{Introduction}

During the last three decades significant work has been done on the subject of noise reduction in image processing with the use of partial differential equations (PDEs). By noise reduction or noise removal we mean the algorithm which has to be applied to a digital image to remove the wrong data information, or artificial imprints added to the digital image by the sensors; while during this procedure it is important that the original information

*Email: anpaliat@phys.uoa.gr 
and structure to be preserved [1. For a review on the various technics on noise reduction methods we refer the reader in [2].

The linear heat equation was one of the first PDEs to which was applied image reduction [3, 4, while the introduction of nonlinear terms into the PDE [5] was necessary in order to avoid the flatness of a noise image. Some other nonlinear models can be found and are presented for instance in [6 9 ].

You and Kaveh in [10] proposed the following nonlinear higher-order diffusion equation 1$]$

$$
\frac{\partial u}{\partial t}=-E_{L}(f|\Delta u|),
$$

where the right hand side of (1) follows from the variational principle of the Action Integral, $S=\int f(|\Delta u|) d \mathbf{x}$. In the case of a two-dimensional flat space this becomes

$$
S=\int f\left(\left|u_{, x x}+u_{, y y}\right|\right) d x d y .
$$

Consequently, $f$ is the Lagrange function, while a second requirement for function $f$ is $f^{\prime}>0$.

Now, in the simplest case for which $f$ is a linear function, i.e. $f(|\Delta u|)=\sqrt{u_{, x x}+u_{, y y}}$, equation (11) takes the following form

$$
\frac{\left(u_{x x x x}+2 u_{x x y y}+u_{y y y y}\right)\left(u_{x x}+u_{y y}\right)-3\left[\left(u_{x x}+u_{y y}\right)_{, x}\right]^{2}-3\left[\left(u_{x x}+u_{y y}\right)_{, y}\right]^{2}}{\sqrt{\left|u_{, x x}+u_{, y y}\right|}}+u_{t}=0 .
$$

That particular linear function, $f$, can be seen as a natural extension of the Lagrangian for the second-order differential equation studied in [5]. While equation (3) is a fourth-order equation, there are similarities with the second-order minimal surface equation. In particular, in the minimal surface equation the Action Integral involves the minimization of a surface and not of an arc length, as is the case with the equations of motion [11-13]. In this work, the equation (3) follows from the minimization of a length which is defined by second-order derivatives.

In this work we study the latter nonlinear fourth-order PDE by applying Lie's theory on the symmetries of differential equations. Lie's theory is one of the main mathematical tools for the determination of solutions for nonlinear differential equations. The existence of a Lie symmetry for a given differential equation is important because invariant functions can be constructed to reduce the order of the differential equation or the number of the dependent variables [14]. Furthermore, Lie symmetries can be used to perform group classifications for differential equations [15] or identify well-known systems for instance see [16 25] and references therein. The applications of Lie symmetries cover various areas of Mathematical physics from liquid films and travel wave solutions [26 29], the theory of diffusion [30,31] and optical physics 32

The application of Lie symmetries to the theory of noise reduction is not new. Recently in [33] a group classification analysis was performed for the nonlinear second-order PDE proposed by Rudin et al [5] and Lie symmetries were applied for the determination of conservation laws. In this work we approach the problem differently, by applying the Lie invariants to perform the reduction process for the PDE (3). The importance of the application of Lie point symmetries on noise reduction PDEs is not only that closed-form solutions can be derived, but Lie symmetries can be applied also to recognize patterns in an image as proposed by Bigün [34,35].

The plan of the paper is as follows.

In Section 2 the basic properties and definitions of Lie point symmetries of differential equations are presented. Moreover, we present the Lie symmetries for equation (3) and that of the time-independent case. The

\footnotetext{
${ }^{1} \Delta$ denotes the Laplace operator.
} 
application of the Lie invariants is performed in Section 3 from where we find that the static solutions reduce always to maximally symmetric second-order differential equations. That is not true for the nonstatic invariant solutions. However, for the latter case we are able to find some particular exact solutions. Finally we discuss our results and draw our conclusions in Section 4.

\section{Lie point symmetries}

Let function $\Phi$ describe the map of an one-parameter point transformation such as $\Phi\left(u\left(x^{i}\right)\right)=u\left(x^{i}\right)$ with infinitesimal transformation

$$
\begin{aligned}
t^{\prime} & =t^{i}+\varepsilon \xi\left(t, x^{i}, u\right) \\
x^{i \prime} & =x^{i}+\varepsilon \xi^{i}\left(t, x^{i}, u\right) \\
u^{\prime} & =u+\varepsilon \eta\left(t, x^{i}, u\right)
\end{aligned}
$$

and generator

$$
\Gamma=\frac{\partial t^{\prime}}{\partial \varepsilon} \partial_{t}+\frac{\partial x^{\prime}}{\partial \varepsilon} \partial_{x}+\frac{\partial u}{\partial \varepsilon} \partial_{u}
$$

where $\varepsilon$ is the parameter of smallness and $x^{i}=(x, y)$.

Let $u\left(x^{i}\right)$ be a solution of the PDE

$$
\mathcal{H}\left(u, u_{, t}, u_{, x} \ldots\right)=0
$$

then under the map $\Phi$, function $u^{\prime}\left(x^{i \prime}\right)=\Phi\left(u\left(x^{i}\right)\right)$ is a solution for the latter differential equation if and only if the differential equation is also invariant under the action of the map, $\Phi$, i.e.

$$
\Phi\left(\mathcal{H}\left(u, u_{, t}, u_{, x} \ldots\right)\right)=0 .
$$

When the latter expression is true, the generator $\Gamma$ is called a Lie point symmetry for the differential equation. It is straightforward to prove that this condition becomes

$$
\Gamma^{[n]}(\mathcal{H})=0
$$

in which $\Gamma^{[n]}$ describes the $n^{t h}$ prolongation/extension of the symmetry vector in the jet-space of variables, $\left\{t, x^{i}, u, u, i, u, i j, \ldots\right\}$.

The importance of the existence of a Lie symmetry for a given PDE is that from the associated Lagrange's system,

$$
\frac{d t}{\xi^{t}}=\frac{d x^{i}}{\xi^{i}}=\frac{d u}{\eta}
$$

zeroth-order invariants, $U^{[0]}\left(t, x^{i}, u\right)$ are able to be determined which can be used to reduce the number of the independent variables of the differential equation and lead to the construction of similarity solutions. Last but not least, the admitted symmetries of a given differential equation constitute a Lie algebra.

\subsection{Lie point symmetries for the time-independent equation}

Consider now $u(t, x, y)=u(x, y)$. Then equation (3) is simplified as follows.

The resulting differential equation is of fourth-order and it is given as

$$
\left(u_{x x x x}+2 u_{x x y y}+u_{y y y y}\right)\left(u_{x x}+u_{y y}\right)-3\left[\left(u_{x x}+u_{y y}\right)_{, x}\right]^{2}-3\left[\left(u_{x x}+u_{y y}\right)_{, y}\right]^{2}=0 .
$$




\begin{tabular}{cccccc}
\hline \hline$[]$, & $X_{1}$ & $X_{2}$ & $X_{3}$ & $X_{4}$ & $X_{5}$ \\
\hline$X_{1}$ & 0 & 0 & $-X_{2}$ & 0 & $X_{1}$ \\
$X_{2}$ & 0 & 0 & $X_{1}$ & 0 & $X_{2}$ \\
$X_{3}$ & $X_{2}$ & $-X_{1}$ & 0 & 0 & 0 \\
$X_{4}$ & 0 & 0 & 0 & 0 & 0 \\
$X_{5}$ & $-X_{1}$ & $-X_{2}$ & 0 & 0 & 0 \\
\hline \hline
\end{tabular}

Table 1: Lie Brackets for the admitted Lie point symmetries of equation (12)

\begin{tabular}{ccccccc}
\hline \hline$[]$, & $Y_{1}$ & $Y_{2}$ & $Y_{3}$ & $Y_{4}$ & $Y_{5}$ & $Y_{6}$ \\
\hline$Y_{1}$ & 0 & 0 & $-Y_{2}$ & 0 & $Y_{1}$ & 0 \\
$Y_{2}$ & 0 & 0 & $Y_{1}$ & 0 & $Y_{2}$ & 0 \\
$Y_{3}$ & $Y_{2}$ & $-Y_{1}$ & 0 & 0 & 0 & 0 \\
$Y_{4}$ & 0 & 0 & 0 & 0 & 0 & $-Y_{6}$ \\
$Y_{5}$ & $-Y_{1}$ & $-Y_{2}$ & 0 & 0 & 0 & $-5 Y_{6}$ \\
$Y_{6}$ & 0 & 0 & 0 & $Y_{6}$ & $5 Y_{6}$ & 0 \\
\hline \hline
\end{tabular}

Table 2: Lie Brackets for the admitted Lie point symmetries of equation (3)

For this time-independent equation the application of Lie's theories provides the following $5+\infty$ Lie point symmetries.

$$
\begin{aligned}
& X_{1}=\partial_{x}, X_{2}=\partial_{y}, X_{3}=y \partial_{x}-x \partial_{x}, X_{4}=u \partial_{u} \\
& X_{5}=x \partial_{x}+y \partial_{y}, X_{\infty}=\Psi(x, y) \partial_{u} \text { with } \Delta \Psi=0 .
\end{aligned}
$$

The Lie Brackets of the admitted Lie symmetries are presented in Table 1 from which it is clear that the admitted Lie algebra is $\left\{3 A_{1} \oplus_{s} 2 A_{1}\right\}$ in the Morozov-Mubarakzyanov Classification Scheme [36 39]. The vector fields $\left\{X_{1}, X_{2}, X_{3}\right\}$ form the $E^{2}$ Lie algebra, more specifically they are the isometries of the two-dimensional Euclidean space, while the set of vectors $\left\{X_{1}, X_{2}, X_{3}, X_{5}\right\}$ form the Homothetic algebra for the two-dimensional Euclidean space.

\subsection{Lie point symmetries for the time-dependent equation}

For the time-dependent equation (3) the admitted Lie point symmetries are calculated to be

$$
\begin{aligned}
& Y_{1}=\partial_{x}, Y_{2}=\partial_{y}, Y_{3}=y \partial_{x}-x \partial_{x}, Y_{4}=t \partial_{t}-2 u \partial_{u}, \\
& Y_{5}=5 t \partial_{t}+x \partial_{x}+y \partial_{y}, Y_{6}=\partial_{t}, \quad Y_{\infty}=\bar{\Psi}(x, y) \partial_{u} \text { with } \Delta \bar{\Psi}=0 .
\end{aligned}
$$

The Lie Brackets of the Lie point symmetries are presented in Table 2 from which we infer that the admitted Lie algebra is $\left\{2 A_{1} \oplus_{s} A_{1}\right\} \oplus_{s} A_{2,1}$.

The table of Lie Brackets is 


\begin{tabular}{ccccc}
\hline \hline$[]$, & $Z_{1}$ & $Z_{2}$ & $Z_{3}$ & $Z_{4}$ \\
\hline$Z_{1}$ & 0 & 0 & $-Z_{2}$ & $Z_{1}$ \\
$Z_{2}$ & 0 & 0 & $Z_{1}$ & $Z_{2}$ \\
$Z_{3}$ & $Z_{2}$ & $-Z_{1}$ & 0 & 0 \\
$Z_{4}$ & $-Z_{1}$ & $-Z_{2}$ & 0 & 0 \\
\hline \hline
\end{tabular}

Table 3: Lie Brackets for the admitted Lie point symmetries of equation (13)

The admitted Lie symmetries are again the isometries of the two-dimensional Euclidean space, but the homothetic field is replaced by the scalar symmetries $\left\{Y_{4}, Y_{5}\right\}$, while also the $Y_{6}$ symmetry follows because the differential equation is invariant under time translations.

\subsection{Lie point symmetries for the time-independent equation with a linear source}

Before we proceed with the application of the Lie invariants, we determine the Lie point symmetries of the following differential equation ,

$$
\left(u_{x x x x}+2 u_{x x y y}+u_{y y y y}\right)\left(u_{x x}+u_{y y}\right)-3\left[\left(u_{x x}+u_{y y}\right)_{, x}\right]^{2}-3\left[\left(u_{x x}+u_{y y}\right)_{, y}\right]^{2}+\lambda \sqrt{\left|u_{, x x}+u_{, y y}\right|} u=0
$$

which is the time-indepedent equation (3) with a linear source $\lambda u$. In the following Section we see how this equation it can follow from (3) for a specific value of the parameter $\lambda$.

The admitted Lie point symmetries are four and they are

$$
\begin{aligned}
& Z_{1}=\partial_{x}, Z_{2}=\partial_{y}, Z_{3}=y \partial_{x}-x \partial_{y} \\
& Z_{4}=x \partial_{x}+y \partial_{y}+10 u \partial_{u} .
\end{aligned}
$$

with Lie Brackets given in Table 3 Easily we can infer that the Lie symmetries form the Lie algebra $\left\{2 A_{1} \oplus_{s} A_{1}\right\} \oplus_{s} A_{1}$.

We proceed with the application of the Lie symmetry vectors for equation (3).

\section{Application of Lie symmetries}

The nonlinear PDE is an $1+2$ fourth-order equation. We continue our analysis by eliminating the timederivative. For that, we start by performing reductions with the use of the vector fields (a) $Y_{6}$ and (b) $Y_{4}$

\subsection{Reduction with $\partial_{t}$}

The application of the autonomous symmetry $Y_{6}$ to equation (3) provides that the solution, $u$, is static, i.e., $u(t, x, y)=u(x, y)$. Consequently the resulting equation is that of expression (12). Now we can continue with the reduction process by using the Lie symmetries $X_{\mathbf{I}}$. 


\subsubsection{Travelling-wave solution}

Consider the vector field $X_{1}+\alpha X_{2}$. Then the resulting Lie invariants are $\zeta=y-\alpha x, u=w(\zeta)$. We consider $\zeta$ to be the new indepedent variable and $w$ the new dependent variable. Thus equation (12) is simplified to the following fourth-order ordinary differential equation (ODE)

$$
\left(a^{2}+1\right)\left(2 \frac{d^{4} w}{d \zeta^{4}} \frac{d^{2} w}{d \zeta^{2}}-3\left(\frac{d^{3} w}{d \zeta^{3}}\right)^{2}\right)=0 .
$$

For this equation easily we can calculate the admitted Lie point symmetries which are

$$
\bar{X}_{1}=\partial_{w}, \bar{X}_{2}=\partial_{\zeta}, \bar{X}_{3}=w \partial_{w}, X_{4}=\zeta \partial_{\zeta} \text { and } X_{5}=\zeta \partial_{w}
$$

which form the Lie algebra $\left\{2 A_{1} \oplus_{s} A_{1}\right\} \oplus_{s} A_{1,1}$.

While we could continue with the application of Lie point symmetries to equation (14), it can easily be integrated and we get

$$
w(\zeta)=w_{1} \ln \left(\zeta-\zeta_{1}\right)+w_{2}\left(\zeta-\zeta_{2}\right)
$$

where $w_{1,2}$ and $\zeta_{1,2}$ are four constants of integration.

Because the parameter $\alpha$ is not involved in the resulting ODE (14) the solution holds also for reduction process of (12) with the vector field $X_{1}$ or $X_{2}$. Moreover, equation (14) can be written easily as the second-order ordinary differential equation

$$
2 W \frac{d^{2} W}{d \zeta^{2}}-3\left(\frac{d W}{d \zeta}\right)^{2}=0
$$

where $W(\zeta)=\frac{d^{2} w}{d \zeta}$. We can easily see that it is maximally symmetric and admits eight Lie point symmetries, which form the $\operatorname{sl}(3, R)$ Lie algebra. Equation (16) can be easily linearised after the transformation $W(\zeta)=$ $(\bar{W}(\zeta))^{-\frac{1}{2}}[4042$.

\subsubsection{Radial solution}

Consider now the application of the Lie symmetry vector $X_{3}$, i.e. of the rotational symmetry. The invariant functions are calculated to be $r=x^{2}+y^{2}$ and $u=h(r)$. The resulting ODE is

$$
-2 \frac{d^{2} h}{d R^{2}}\left(\frac{d^{4} h}{d R^{4}}+\frac{d^{3} h}{d R^{3}}\right)+\left(\frac{d^{2} h}{d R^{2}}\right)^{2}+3\left(\frac{d^{3} h}{d R^{3}}\right)^{2}=0
$$

after we applied the transformation $r=\exp (R)$ in order to simplify the reduced equation.

Equation (17) admits the four-dimensional Lie algebra, $2 A_{1} \oplus_{s} A_{1,1}$, which comprises the following symmetry vectors

$$
X_{1}^{\prime}=\partial_{R}, X_{1}^{\prime}=\partial_{h}, X_{3}^{\prime}=h \partial_{h} \text { and } X_{4}=R \partial_{h} .
$$

The fourth-order ODE can be easily reduced to the second-order ODE

$$
-2 \rho\left(\frac{d^{2} \rho}{d R^{2}}+\frac{d \rho}{d R}\right)+\rho^{2}+3\left(\frac{d \rho}{d R}\right)^{2}=0
$$

by applying the change of variable $\rho=\frac{d^{2} h}{d R^{2}}$. 
Surprisingly, equation (18) is maximally symmetric, that is, it admits as Lie point symmetries the elements of the $s l(3, R)$ Lie algebra in the representation

$$
\begin{aligned}
& \partial_{R}, \rho \partial_{\rho}, e^{-\frac{R}{2}} \rho^{\frac{3}{2}} \partial_{\rho}, R e^{-\frac{R}{2}} \rho^{\frac{3}{2}} \partial_{\rho}, R \partial_{R}+R \rho \partial_{\rho}, \\
& \frac{e^{\frac{\rho}{2}}}{\sqrt{\rho}}\left(\partial_{R}+\rho \partial_{\rho}\right), \frac{e^{\frac{\rho}{2}}}{\sqrt{\rho}}\left(R \partial_{R}+\rho(R-2) \partial_{\rho}\right), R^{2} \partial_{R}+R(\rho R-2 \rho) \partial_{\rho} .
\end{aligned}
$$

The generic solution of (18) is given to be

$$
\rho(R)=\rho_{0} e^{R}\left(\left(R-R_{0}\right)\right)^{-2},
$$

where $\rho_{0}, R_{0}$ are constants of integration.

\subsubsection{Scaling solution}

From the scaling symmetry $X_{5}$ we determine the invariants $\theta=\arctan \left(\frac{y}{x}\right)$ and $u=g(\theta)$. The reduced ODE is

$$
2\left(\frac{d^{2} g}{d \theta^{2}}\right)\left(\frac{d^{4} g}{d \theta^{4}}\right)-3\left(\frac{d^{3} g}{d \theta^{3}}\right)^{2}-4\left(\frac{d^{2} g}{d \theta^{2}}\right)^{2}=0
$$

which is invariant under the Lie symmetry vectors

$$
X_{1}^{*}=\partial_{\theta}, X_{2}^{*}=\partial_{g}, X_{3}^{*}=g \partial_{g} \text { and } X_{4}^{*}=\theta \partial_{g} .
$$

The generic solution of equation (20) easily can be determined. It is

$$
g(\theta)=g_{1} \ln \left(\cos \left(\theta-\theta_{1}\right)\right)+g_{2}\left(\theta-\theta_{2}\right)
$$

in which $g_{0,1}$ and $\theta_{0,1}$ are four constants of integration.

However, what is important to mention is that equation (20) can be written again as a second-order ODE by applying the change of variable $G=\left(\frac{d^{2} g}{d \theta^{2}}\right)$. The resulting second-order ODE is maximally symmetric and under the $G=\bar{G}^{-2}$ takes the simple form of the harmonic oscillator

$$
\frac{d^{2} \bar{G}}{d \theta^{2}}+\bar{G}=0
$$

\subsection{Reduction with $t \partial_{t}-2 u \partial_{u}$}

We continue our analysis by applying the invariants to equation (3) of the symmetry vector $Y_{4}$. The invariant functions are $x, y$ and $u=\frac{u(x, y)}{t^{2}}$. The resulting PDE is equation (13) for the specific value $\lambda=-2$.

\subsubsection{Travelling-wave solution}

Reduction with the use of the symmetry vectors $Z_{1}+\alpha Z_{2}$ provides the fourth-order ODE

$$
2\left(\frac{d^{2} w}{d \zeta^{2}}\right)\left(\frac{d^{4} w}{d \zeta^{4}}\right)-3\left(\frac{d^{3} w}{d \zeta^{3}}\right)^{2}-2 \sqrt{\left(\alpha^{2}+1\right)^{-5} \frac{d^{2} w}{d \zeta^{2}}} w=0
$$

in which $\zeta=y-\alpha x$ and $w=w(\zeta)$.

Equation (23) is invariant only under the two-dimensional Lie algebra $A_{1,1}$ with as elements the vector fields

$$
\bar{Z}_{1}=\partial_{\zeta}, \bar{Z}_{2}=\zeta \partial_{\zeta}+10 w \partial_{w}
$$


A special solution of (23) can be found easily by the use of the symmetry vector $\bar{Z}_{2}$ and it is $w(\zeta)=w_{0} \zeta^{10}$ for $w=w(\alpha)$.

The application of the two symmetries $\bar{Z}_{1}, \bar{Z}_{2}$ to (23) leads to a second-order equation with no Lie point symmetries.

\subsubsection{Radial solution}

From the vector field $Z_{3}$ we get the invariants $R=\ln \left(x^{2}+y^{2}\right)$ and $u=h(r)$. The reduced ODE is

$$
-2 \frac{d^{2} h}{d R^{2}}\left(\frac{d^{4} h}{d R^{4}}+\frac{d^{3} h}{d R^{3}}\right)+\left(\frac{d^{2} h}{d R^{2}}\right)^{2}+3\left(\frac{d^{3} h}{d R^{3}}\right)^{2}-\frac{1}{16} \sqrt{\left(\frac{d^{2} h}{d R^{2}}\right)} h e^{\frac{5}{2} R}=0,
$$

which admits only one Lie point symmetry, namely

$$
Z^{\prime}=\partial_{R}+5 h \partial_{h}
$$

Application of this to (24) leads to a third-order ODE with no Lie point symmetries. A special solution of (24) can be found by applying the invariant of $Z^{\prime}$. It is $h(R)=h_{0} e^{5 R}$, where $h_{0}$ is an imaginary number.

\subsubsection{Scaling solution}

The application of the scaling symmetry, $Z_{4}$, leads to a fourth-order ODE with only one symmetry, the vector field $Z^{*}=Z_{3}$ the application of which reduces the equation to a third-order equation without Lie point symmetries. For convenience of the presentation we do not write the fourth-order or the third-order equations.

\section{Conclusions}

In this work, we have focused on the application of Lie's theory for the determination of invariant one-parameter point transformations for a fourth-order $1+2$ evolution equation which has been proposed for the study of noise reduction in image-processing theory. The model of our consideration is a higher-order generalization of the model proposed by Rudin et al [5] and it has been proposed later by You and Kaveh [10].

We have performed the symmetry classification for the following kind of solutions: (i) time-dependent solution, (ii) stationary solution with no source and (iii) stationary solution with linear source. The later classification has been useful in order to continue the reduction process on the $1+2$ evolution equation to a fourth-order ODE.

The line point symmetries have been applied in order to perform second-reductions that they provide similarity solutions which belong to family of: travelling-wave solutions, radial solutions and scaling solutions. Surprisingly, in the case of static solutions all the second-reductions reduce to fourth-order ODEs which can be written as linear second-order ODEs. That it is not the case of nonstatic solutions for which we were able to reduce the fourth-order ODEs to third-order ODEs with the application of Lie symmetries. However, some specific closed-form solutions were determined.

It is important to mention the existence of the two symmetries $u \partial_{u}$ and $\Psi(x, y) \partial_{u}$ which indicates linearity, even if the PDE (3) is always nonlinear. These two symmetries are related with the solution of the twodimensional Laplace equation which is the common factor in (3). However, these solutions are not acceptable for our consideration. For that reason we have not applied these two symmetries in the reduction process. On 
the other hand, from the similarity solutions we determined with the use of the Lie symmetries we show that new solutions exist.

The results of this work indicates that the theory of symmetries of differential equations can be play an important role for the study of the nonlinear PDEs in the image-processing theory. Therefore, conservation laws can be also determined by using Noether's theorem or by using other approaches. Such a work is still in progress and will be published elsewhere.

\section{Acknowledgements}

PGLL Thanks the Durban University of Technology, the University of KwaZulu-Natal and the National Research Foundation of South Africa for support. The authors thank Suranaree University of Technology, Sergey Meleshko and Eckart Schulz for the the hospitality provided while the bulk of this work undertaken.

\section{References}

[1] D. Marr and E. Hildreth, Proc. Roy. Soc. London Ser. B 207, 187 (1980)

[2] P. Bouboulis, K. Slavakis and S. Theodoridis, IEEE Transactions on Image Processing 19, 1465 (2010)

[3] F. Catte, P.-L. Lions, J.-M. Morel and T. Coll, SIAM J. Numer. Anal. 29, 182 (1992)

[4] J. Weickert, Anisotropic diffusion in image processing, Teubner, Stuttgart, Germany, (1998)

[5] L.I. Rudin, S. Osher and E. Fatemi, Physica D 60, 259 (1992)

[6] S.J. Oscher and J.A. Sethian, J. Comput. Phys. 79, 12 (1988)

[7] C. Vogeland M. Oman, Siam J. Sci. Statist. Comput. 17, 227 (1996)

[8] R.T. Whitaker and S.M. Pizer, Comput. Vis. Graph. Image Process.: Image Understand, 57, 99 (1993)

[9] T. Liu and Z. Xiang, J. Math. Probl. Eng. 2013, 1 (2013)

[10] Y.-L. You, and M. Kaveh, IEEE Transactions on Image Processing 9, 1723 (2000)

[11] N. Bila, Lie Groups Applications to Minimal Surfaces PDE, Proceedings of the Workshop on Global Analysis, Differential Geometry and Lie Algebras, BSG Proceedings 3 (1999), G. Tsagas (ed.), Geometry Balkan Press, 196.

[12] A. Peterson and S. Taylor, Locally Isometric Families of Minimal Surfaces, Balkan Journal of Geometry and Its Applications 13, 80 (2008)

[13] A. Aslam and A. Qadir, Noether Symmetries of the Area-Minimizing Lagrangian, J. Applied Mathematics $2012532690(2012)$

[14] G.W. Bluman and S. Kumei, Symmetries of Differential Equations, Springer-Verlag, New York, (1989)

[15] L. V. Ovsiannikov, Group analysis of differential equations, Academic Press, New York, (1982)

[16] P.G.L. Leach, J. Phys. A: Math. Gen. 13, 1991 (1980) 
[17] F.M. Mahomed and P.G.L. Leach, Quaestiones Mathematicae 12, 121 (1989)

[18] M. Tsamparlis and A. Paliathanasis, J. Phys. A: Math. Theor. 45, 275202 (2012)

[19] V.M. Boyko, R.O. Popovych and N.M. Shapoval, J. Math. Anal. Appl. 397, 434 (2013)

[20] F.M. Mahomed and P.G.L. Leach, J. Math. Anal. Appl. 151, 80 (1990)

[21] F.M. Mahomed, Math. Meth. Appl. Sci. 30, 1995 (2007)

[22] A. Ahmad, A.H. Bokhari, A.H. Kara and F.D. Zaman, Quaestiones Mathematicae 33, 75 (2010)

[23] A.H. Kara and F.M. Mohamed, J. Nonl. Math. Phys. 9, 60 (2002)

[24] A. Paliathanasis, M. Tsamparlis and M.T. Mustafa, Comm. Nonl. Sci. Num. Sim. 55, 68 (2018)

[25] P.G.L. Leach, R.R. Warne, N. Caister, V. Naicker and N. Euler, Proccedings: Mathematical Sciences 120, $113(2010)$

[26] X. Xin, H. Liu, L. Zhang and Z. Wang, Appl. Math. Lett. 88, 132 (2019) cl8,cl9,c10,cl11

[27] H. Liu, Z. Wang and X. Xin, Commun. Theor. Phys. 70, 014 (2018)

[28] X. Xin, Y. Liu and X. Liu, Appl. Math. Lett. 55, 63 (2016)

[29] F. Tchier, M. Inc and A. Yusuf, EPJ Plus 134, 250 (2019)

[30] A. Yusuf, M. Inc and M. Bayram, Phys. Scr. 94, 125005 (2019)

[31] M. Inc, A. Yusuf, A.I. Aliyu and D. Baleanu, Optical and Quantum Electronics 50, 94 (2018)

[32] A.I. Aliyu, M. Inc, A. Yusuf, M. Bayram and D. Baleanu, Mod. Phys. Lett. B 33, 1950035 (2019)

[33] B. Al Qurashi, A.H. Kara and H. Akca, Acta Appl. Math. 153, 163 (2018)

[34] J. Bigün, Computer Vision and Image Understanding 68, 290 (1997)

[35] J. Bigün, Local Symmetry Features in Image Processing, Linköping Studies in Science and Technology, Dissertations No. 179, Linköping (1989)

[36] Morozov VV (1958), Izvestia Vysshikh Uchebn Zavendeniı Matematika, 5 161-171

[37] Mubarakzyanov GM (1963), Izvestia Vysshikh Uchebn Zavendeniu Matematika, 32 114-123

[38] Mubarakzyanov GM (1963), Izvestia Vysshikh Uchebn Zavendeniu Matematika, 34 99-106

[39] Mubarakzyanov GM (1963), Izvestia Vysshikh Uchebn Zavendeniu Matematika, 35 104-116

[40] M. Aguirre, J. Math. Phys. 29, 1746 (1988)

[41] F.M. Mahomed and P.G.L. Leach, Quaestiones Mathematicae, 12, 121 (1989)

[42] V.M. Gorringe and P.G.L. Leach, Quaestiones Mathematicae, 11, 95 (1988) 